GREGORY W. RUTECKI, MD, Section Editor

Marc Ohlhausen, BS

Medical Student, Case Western Reserve

School of Medicine, Cleveland, $\mathrm{OH}$
Eden Bernstein, MD

Internal Medicine Resident, Department of Internal Medicine, Cleveland Clinic,

Cleveland, $\mathrm{OH}$
Craig D. Nielsen, MD, FACP

Department of Internal Medicine, Cleveland Clinic; Associate Professor, Cleveland Clinic Lerner College of Medicine of Case Western Reserve University, Cleveland, $\mathrm{OH}$; Governor, Ohio Chapter, American College of Physicians; Deputy Editor, Cleveland Clinic Journal of Medicine

\title{
An 86-year-old man with unexplained right-sided headache and vision loss
}

If giant cell arteritis is strongly suspected, high-dose systemic glucocorticoids should be started promptly
A N 86-yeAR-OLD man presented to a local hospital ophthalmologist with headache and pain in his right temple without vision loss. Laboratory values for complete blood cell count, erythrocyte sedimentation rate (ESR), and $\mathrm{C}$-reactive protein (CRP) were normal. The medical history was remarkable only for a remote history of diverticulitis. He reported social consumption of alcohol and smoking in the past.

Although reassured, the patient returned 2 weeks later with acute loss of vision in his right eye, preceded by eye discomfort, floaters, flashing lights, and worsening right temporal pain and headache. On physical examination, he had no fever and was normotensive. Extraocular movements and visual fields were normal, and visual acuity was unchanged. Heart, lung, joint, and skin examinations were unremarkable.

Given the patient's age and presentation, giant cell arteritis (GCA) was suspected. Key clinical features of GCA in patients over age 50 include abrupt new headache, scalp pain and tenderness, jaw claudication, visual symptoms, polymyalgia rheumatica, temporal artery abnormalities, and elevated ESR or CRP, or both. ${ }^{1,2}$ Anterior ischemic optic neuropathy due to occlusion of the posterior ciliary artery is the cause in $85 \%$ of cases of vision loss in GCA. ${ }^{3}$

\section{EVALUATING AND MANAGING SUSPECTED GIANT CELL ARTERITIS}

What is the most appropriate first step for a patient with suspected GCA?

Oral glucocorticoid therapy

Biopsy of the temporal artery

Measurement of serum ESR and CRP

Duplex ultrasonography of the temporal artery

Ophthalmologic assessment

If GCA is strongly suspected, high-dose systemic glucocorticoids should be started promptly to prevent irreversible vision loss and involvement of the other eye. ${ }^{4}$ Urgent referral for specialist management, ophthalmologic assessment, and temporal artery biopsy are recommended but should not delay administration of glucocorticoids. Temporal artery biopsy is the preferred method of confirming GCA, although a negative result does not rule out disease in cases of high clinical suspicion. ${ }^{1}$

Initiating glucocorticoids should lead to significant improvement in symptoms. If this does not occur, one should evaluate for alternative diagnoses.

\section{CASE CONTINUED: SYMPTOMS PROGRESS DESPITE STEROIDS}

The patient was started on oral prednisone 60 mg for presumed GCA and admitted to the local hospital. ESR and CRP were repeated, and results again were within normal range. A right-sided temporal artery biopsy was per- 


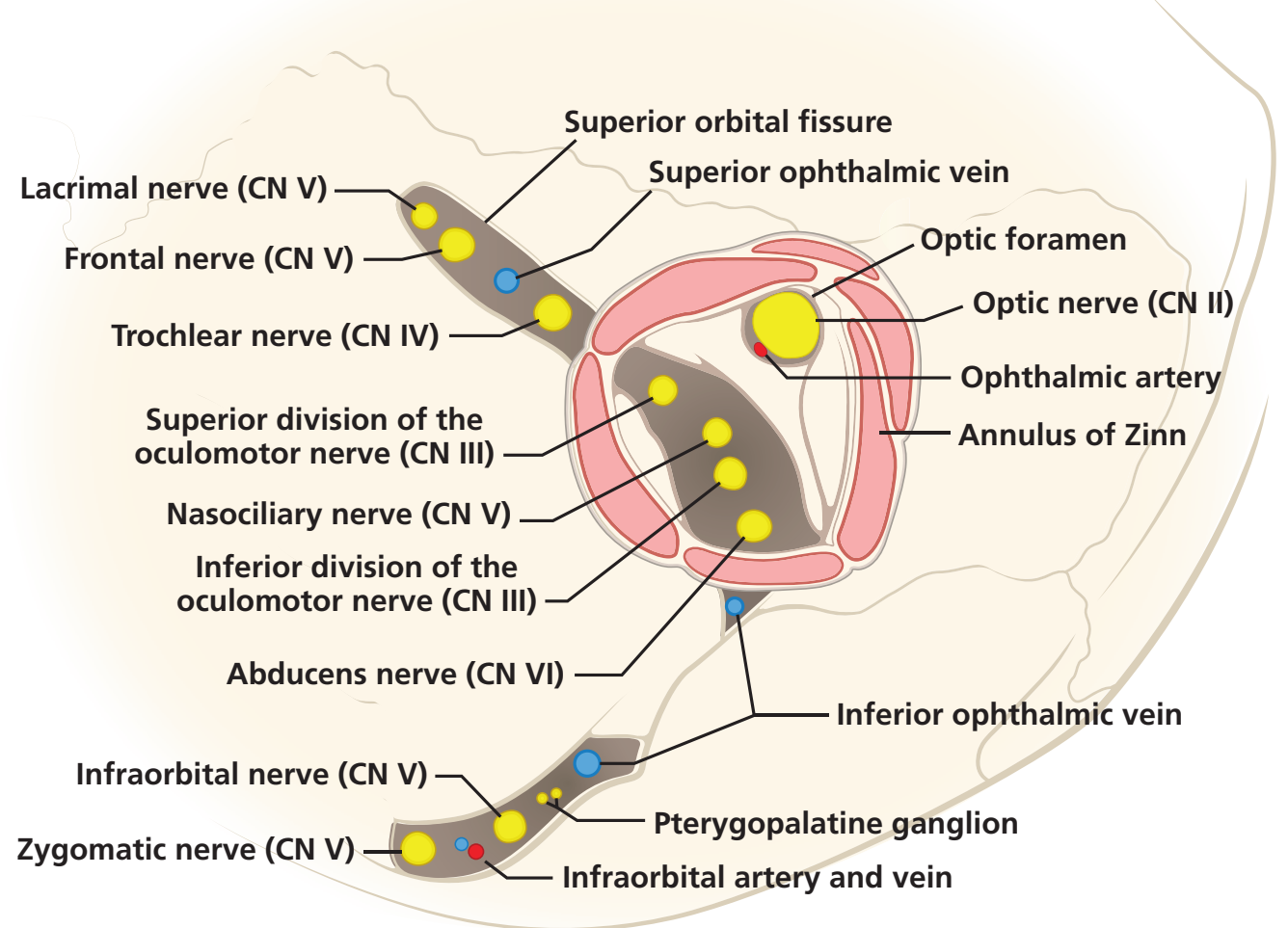

Figure 1. The anatomy of the orbital apex, the most posterior aspect of the orbit. The unilateral cranial nerve $(\mathrm{CN})$ deficits in this patient point to disruption of structures of the orbital apex including the optic, oculomotor, trochlear, and abducens nerves. The optic foramen contains the optic nerve, ophthalmic artery, and associated sympathetic nerves. The nasociliary, frontal, and lacrimal branches of the ophthalmic nerve, superior ophthalmic vein, and cranial nerves III, IV, and VI pass through the superior orbital fissure.

formed but showed no evidence of active or healed arteritis. Magnetic resonance imaging (MRI) and computed tomography (CT) angiographic imaging of the head and neck were unremarkable.

The patient was discharged. The diagnosis of GCA was considered unlikely, but prednisone was continued out of concern for possible vision loss in the unaffected eye while other causes were being evaluated. Prednisone was up-titrated to $70 \mathrm{mg}$ due to lack of symptom improvement, with no improvement in vision or headache severity reported.

The patient was referred to the ophthalmology department at a nearby large academic hospital for further workup. On presentation 1 month after his hospitalization, a variety of new signs and symptoms had developed. Ophthalmologic examination revealed right-sided ptosis, eyelid swelling, and chemosis (ie, swelling of the conjunctiva). The right eye was unable to gaze superiorly, inferiorly, or laterally. Vision in the right eye was completely absent, with no light perception. The right pupil was nonreactive to light, and an afferent pupillary defect was present. The fundus appeared normal on dilated fundus examination. Intraocular pressures were $20 \mathrm{~mm}$ $\mathrm{Hg}$ (reference range $12-22 \mathrm{~mm} \mathrm{Hg}$ ) in both eyes. Tenderness to palpation was noted over the right eyebrow, temple, and forehead. All findings in the left eye were normal.

The patient was admitted for additional workup. Initial laboratory investigations revealed a white blood cell count of $10.47 \times$ $10^{9} / \mathrm{L}$ (reference range 3.70-11.00 × 10\% $/ \mathrm{L}$ ) with $90.9 \%$ neutrophils, $5.9 \%$ lymphocytes, $3.1 \%$ monocytes, $0 \%$ eosinophils, and $0.1 \%$ basophils. A complete metabolic panel was within normal limits except for an elevated blood glucose level of $170 \mathrm{mg} / \mathrm{dL}$ (reference range 74-99 $\mathrm{mg} / \mathrm{dL}$ ), which was attributed
The orbital apex is the posteriormost end of the orbit and comprises bony, neural, and vascular structures 
to steroid therapy. The ESR was $4 \mathrm{~mm} /$ hour (reference range $<30 \mathrm{~mm} /$ hour) and CRP was $0.15 \mathrm{mg} / \mathrm{dL}$ (reference range $<0.30 \mathrm{mg} / \mathrm{dL}$ ).

Given the lack of response to prednisone, the normal ESR and CRP levels, and the negative temporal artery biopsy, the diagnosis of GCA was ruled out, and evaluation for other causes continued.

\section{CRANIAL NERVE PATTERN PROVIDES CLUES}

2

Involvement of the neural structures at which of these locations best explains the pattern of cranial nerve deficits seen on this patient's examination?
Orbital apex
Cavernous sinus
Optic nerve
Retina
Superior orbital fissure

The pattern of neurologic deficits localizing unilaterally to cranial nerves II (optic), III (oculomotor), IV (trochlear), and VI (abducens) seen in this patient is most consistent with disruption of structures at the orbital apex. ${ }^{5}$ The orbital apex is the posterior-most end of the orbit and is made up of bony, neural, and vascular structures (Figure 1). Within the orbital apex are 2 orifices in the sphenoid bone:

- The optic foramen, which contains the optic nerve, the ophthalmic artery, and associated sympathetic nerves

- The superior orbital fissure, a bony cleft lateral to the optic foramen, through which pass the nasociliary, frontal, and lacrimal branches of the ophthalmic nerve $\left(\mathrm{V}_{1}\right.$, ie, first division of cranial nerve $\mathrm{V}$ [trigeminal]), superior ophthalmic vein, and cranial nerves III, IV, and VI.

The annulus of Zinn is the common tendinous origin of the recti muscles and surrounds the optic foramen and the central portion of the superior orbital fissure. The contents of this annulus are the optic nerve, ophthalmic artery, oculomotor nerve, abducens nerve, and nasociliary nerve. Because of their confinement, these structures are at greater risk of compression or shear injury. ${ }^{6}$

The presence of multiple nerve palsies in this patient's presentation indicated that his condition was unlikely due to a primary pathology at the retina or optic nerve. ${ }^{7}$ In addition, on dilated fundus examination there was no optic nerve pallor and no finding suggestive of central retinal artery occlusion or retinal detachment.

3 Which of these syndromes is most likely to 3 cause optic nerve dysfunction?

Orbital apex syndrome

Cavernous sinus syndrome

Superior orbital fissure syndrome

Rochon-Duvigneaud syndrome

Orbital apex syndrome is the constellation of signs and symptoms resulting from a disease process affecting the orbital apex structures characterized by involvement of cranial nerves II, III, IV, VI, and $\mathrm{V}_{1}$. The most common presenting features are vision loss, ophthalmoplegia, and blurred vision. ${ }^{8,9}$ Involvement of the oculomotor, abducens, and trochlear nerves causes ophthalmoplegia and diplopia owing to disruption of innervation to the extraocular muscles. Oculomotor nerve palsy also causes ipsilateral ptosis and mydriasis. Involvement of cranial nerve $V_{1}$ results in hypoesthesia or pain of the ipsilateral forehead and upper eyelid, along with absence of corneal reflex and sensation. Inflammation due to infectious, inflammatory, or neoplastic processes may cause proptosis. Variations in presentation of orbital apex syndrome are common owing to the large number of structures involved.

Due to close anatomic proximity, overlapping clinical features are found in two additional syndromes: cavernous sinus syndrome and superior orbital fissure syndrome (also known as Rochon-Duvigneaud syndrome). ${ }^{8-11}$ The names of these three syndromes correlate with the anatomic location of their disease processes.

Cavernous sinus syndrome presents similarly to orbital apex syndrome but also involves the maxillary nerve $\left(\mathrm{V}_{2}\right)$. This results in hypoesthesia of the cheek and lower eyelid, along with facial pain extending farther inferiorly than the periorbital region innervated by the ophthalmic branch. ${ }^{10}$ On examination, the most useful distinction between cavernous sinus syndrome and orbital apex syndrome is involvement of the optic nerve, which is rare 
in cavernous sinus syndrome. ${ }^{11}$ Also of note, cavernous sinus syndrome may cause Horner syndrome (manifested by ptosis, miosis, and anhidrosis) due to the involvement of the sympathetic chain adjacent to the cavernous segment of the internal carotid artery. Vascular etiologies of cavernous sinus syndrome are classically associated with a pulsatile proptosis. ${ }^{8}$

Superior orbital fissure syndrome occurs with a lesion directly anterior to the orbital apex, affecting structures coursing through the superior orbital fissure at this location. The presentation is similar to that of orbital apex syndrome but without optic nerve impairment. Superior orbital fissure syndrome can be progressive, and patients may go on to develop orbital apex syndrome or cavernous sinus syndrome. ${ }^{8}$

\section{EVALUATING THE CAUSE OF ORBITAL APEX SYNDROME}

Possible causes of orbital apex syndrome are numerous and varied (Table 1 ) $^{8,10}$ In the absence of recent surgery or trauma, inflammatory, infectious, vascular, and neoplastic etiologies must be considered.

Tolosa-Hunt syndrome is a common inflammatory cause of orbital apex syndrome. It presents with periorbital pain and limited eye movements, most often unilaterally.

Immunoglobulin G4-related disease is a systemic inflammatory condition that can include salivary gland enlargement, lymphadenopathy, retroperitoneal fibrosis, and pancreatitis. Ocular involvement most commonly includes chronic lid swelling and proptosis, but may include visual disturbances from orbital apex syndrome. ${ }^{12}$

Both Tolosa-Hunt syndrome and immunoglobulin G4-related disease have characteristic findings on neuroimaging and generally respond to steroids.

Vasculitides associated with antineutrophil cytoplasmic antibody (ANCA) (eg, granulomatosis with polyangiitis, microscopic polyangiitis, and eosinophilic granulomatosis with polyangiitis) generally present with pulmonary, gastrointestinal, and neurological involvement but can also have ocular involvement like orbital apex syndrome, as well as corneal ulceration, episcleritis, scleritis, and

\section{TABLE 1}

\section{Causes of orbital apex syndrome}

\section{Inflammatory}

Sarcoidosis, systemic lupus erythematosus, antineutrophil cytoplasmic antibody vasculitis (granulomatosis with polyangiitis, eosinophilic granulomatosis with polyangiitis, microscopic polyangiitis), TolosaHunt syndrome, giant cell arteritis, orbital inflammatory pseudotumor, thyroid orbitopathy, immunoglobulin G4-related disease

\section{Infectious}

Fungi: Aspergillosis, mucormycosis

Bacteria: Streptococcus species, Staphylococcus species, Actinomyces species, gram-negative bacilli, anaerobes, Mycobacterium tuberculosis

Spirochetes: Treponema pallidum

Viruses: Herpes zoster ophthalmicus

\section{Neoplastic}

Head and neck tumors: nasopharyngeal carcinoma, adenoid cystic carcinoma, squamous cell carcinoma

Neural tumors: neurofibroma, meningioma, ciliary neurinoma, schwannoma

Metastases: lung, breast, renal cell, malignant melanoma

Hematologic: non-Hodgkin lymphoma, leukemia

Perineural invasion of cutaneous malignancy

\section{latrogenic}

Sinonasal surgery, orbital-facial surgery

\section{Traumatic}

Orbital apex fracture, retained foreign body, penetrating or nonpenetrating injury

\section{Vascular}

Carotid cavernous aneurysm, carotid cavernous fistula, cavernous sinus thrombosis, sickle cell anemia

\section{Other}

Mucocele

retinal vascular occlusion.

Sarcoidosis is a granulomatous inflammatory disease that primarily affects the lungs. The most common ocular involvement of sarcoidosis is anterior uveitis, although cases of sarcoid granulomas eroding into the orbital apex have been reported. ${ }^{13}$

Because immunosuppressive therapy is indicated for inflammatory causes, infectious etiologies should first be considered so as not to exacerbate them with treatment. 

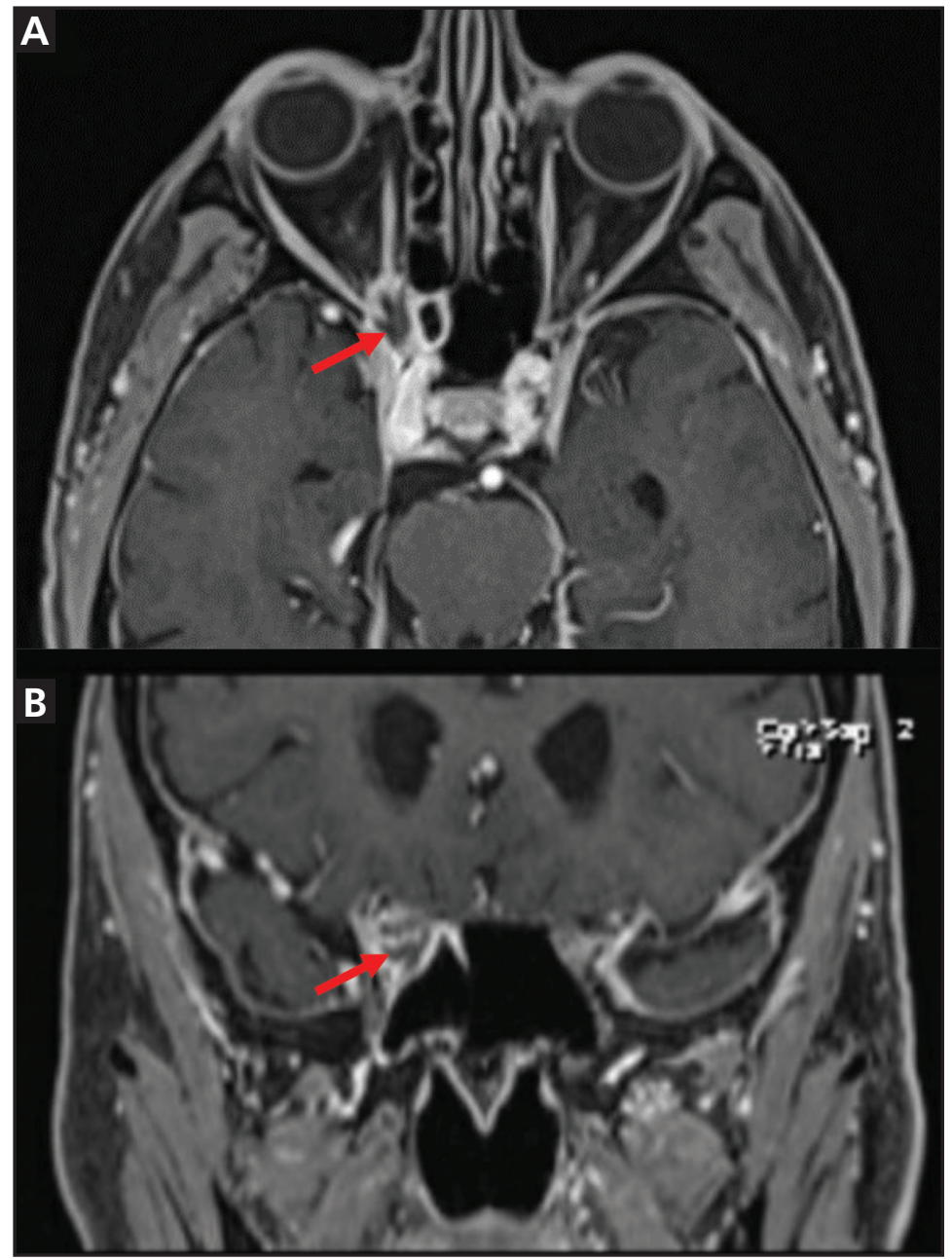

Figure 2. Magnetic resonance imaging of the orbit showed lesions (arrows) in axial T1-weighted fat-suppressed series (A) and coronal multiplanar reformation (B) views.

Bacterial and fungal infections of the paranasal sinuses can spread to the contiguous orbital apex. Fungal infections are primarily found in immunocompromised patients.

Neoplastic etiologies include meningeal infiltration of leukemia or lymphoma, as well as nasopharyngeal carcinoma, which portends a poor prognosis. ${ }^{14}$

\section{CASE CONTINUED: FURTHER EVALUATION}

A serologic vasculitis workup including ANCA, antinuclear antibodies, and doublestranded DNA antibodies was negative. Human immunodeficiency virus testing was negative. Lumbar puncture was performed to evaluate for evidence of inflammatory, infec- tious, and neoplastic processes in the central nervous system. Cerebrospinal fluid analysis showed no white blood cells, normal protein, elevated glucose $(122 \mathrm{mg} / \mathrm{dL}$, reference range $45-80 \mathrm{mg} / \mathrm{dL}$ ), and no growth on cultures.

\section{IMAGING THE ORBITAL APEX}

What is the preferred imaging method for evaluating lesions of the orbital apex?

\section{MRI \\ CT \\ Ultrasonography \\ Radiography}

High-resolution MRI with and without contrast is preferred for evaluating most lesions of the orbital apex. ${ }^{8}$ MRI provides superior softtissue contrast compared with other imaging methods, and orbital fat suppression can also improve lesion visibility. ${ }^{15} \mathrm{~A}$ short-tau inversion recovery image or $\mathrm{T} 2$-weighted fat-suppressed series can be included to evaluate for inflammatory edema and purulent fluid collections. ${ }^{16}$

CT is useful for evaluating bone involvement at the orbital apex, especially in the setting of trauma. ${ }^{10} \mathrm{It}$ is also used for patients with magnetic foreign bodies, surgical clips, or other MRI contraindications.

\section{CASE CONTINUED: DIAGNOSTIC IMAGING AND BIOPSY}

Brain MRI revealed an irregularly shaped lesion with peripheral enhancement and a central nonenhancing region just inferior to the right optic nerve at the orbital apex (Figure $2)$. It was thought that this finding might represent a small abscess or area of necrosis. Scattered paranasal sinus mucosal thickening and increasing asymmetric enlargement of the right anterior cavernous sinus relative to previous images were also noted.

Sinus CT showed corresponding heterogeneous soft tissue at the right orbital apex with smooth bony remodeling and subtle erosive changes, raising suspicion for a neoplasm, infection, or an inflammatory entity. There appeared to be thinning and convexity of the right sphenoid sinus roof in addition to erosion of the right optic strut and along the inferior margin of the right anterior clinoid process. 
An endoscopic transnasal intracranial biopsy was performed. An incision made within the right orbital apex inferior to the optic nerve returned purulent material. Frozen sections from the right orbital apex were negative for neoplasm but showed invasive fungal hyphae within fragments of fibrous tissue with focal necrosis. On Grocott-Gomori methenamine silver stain, the organism morphologically resembled aspergillus species. Aspergillus fumigatus was confirmed by fungal culture.

The patient was then diagnosed with invasive orbital aspergillosis with involvement of the orbital apex and cavernous sinus.

\section{ORBITAL FUNGAL INFECTIONS}

5 Which pair of organisms most commonly cause orbital mycoses?

\section{Aspergillus and Mucor}

Mucor and Candida

Aspergillus and Candida

Candida and Fusarium

Aspergillosis and mucormycosis are the most common causes of orbital fungal infections. ${ }^{17}$

Candida species are the most common etiologic pathogens of keratitis. Fusarium species are also one of the predominant causes of corneal fungal infections and are the most likely fungal pathogen to cause infection following eye trauma. ${ }^{18}$

Orbital mycoses are most often a result of contiguous spread from the paranasal sinuses. ${ }^{10}$

Rhizopus species are the most common cause of mucormycosis, which is classically associated with diabetes. ${ }^{19,20}$

\section{Orbital aspergillosis}

A flavus and A fumigatus are the most common species of aspergillus that affect the orbit. ${ }^{21}$ Orbital aspergillosis presents in invasive and noninvasive forms.

Noninvasive aspergillosis typically does not display fungal infiltration of tissue but can produce a thick material known as allergic mucin along with extramucosal mycotic proliferations known as fungus balls, which are seen primarily in patients who are immunocompetent. If located in the posterior sphenoid or ethmoid sinus, a large fungus ball may compress the optic nerve and cause vision loss and even orbital apex syndrome..$^{22}$

Invasive aspergillosis is characterized by bone invasion and fungal tissue that behaves similarly to an inflammatory or malignant process. ${ }^{23}$ Locally, there is invasion of nearby structures and blood vessels, causing thrombosis and tissue necrosis. In the fulminant form of invasive aspergillosis, there is embolization and multiorgan involvement, potentially leading to death. ${ }^{21}$ Risk factors for invasive aspergillosis include total neutrophil count of less than $1,000 / \mathrm{mm}^{3}$, T-cell defects (eg, from human immunodeficiency virus), defective phagocytosis, hematologic malignancy, immunosuppressive agents, diabetes mellitus, prosthetic devices, trauma, excessive environmental exposure, residence in an endemic area (eg, Sudan), and advanced age. ${ }^{20}$ While incidence of invasive disease is much greater in immunocompromised patients, cases have also been reported in immunocompetent hosts. ${ }^{21-30}$

\section{TREATING INVASIVE ASPERGILLOSIS}

6 Which antifungal medication is preferred 0 for the initial treatment of invasive aspergillosis?

\section{Amphotericin B deoxycholate \\ Voriconazole \\ Isavuconazole \\ Echinocandins}

The Infectious Diseases Society of America 2016 update of practice guidelines for diagnosis and management of aspergillosis recommends voriconazole for initial medical therapy for invasive sinus aspergillosis. ${ }^{30}$ For patients who are intolerant to voriconazole, the best alternative is a lipid formulation of amphotericin B or isavuconazole. Treatment is recommended for a minimum of 6 to 12 weeks, depending on the degree of immunosuppression, infection location, and evidence of improvement.

Lipid formulations of amphotericin B are less likely to cause nephrotoxicity compared with amphotericin B deoxycholate. Amphotericin $\mathrm{B}$ deoxycholate is not recommended for use in invasive aspergillosis unless lipid formulations of amphotericin or other moldactive antifungals (such as voriconazole) are
High-resolution

MRI with and without contrast is preferred for evaluating most lesions of the orbital apex 
unavailable. ${ }^{30}$ Hyperbaric oxygen and retrobulbar amphotericin B injections are less commonly used to treat orbital mycoses, but there is some evidence for their viability. ${ }^{31-33}$ Retrobulbar injections may be useful if aggressive orbital debridement is not favored or if the burden of orbital disease is not substantial. ${ }^{33}$

In general, combination therapy is not recommended, but the use of voriconazole with an echinocandin may be considered for patients with severe disease, hematologic malignancy, or profound persistent neutropenia. ${ }^{30}$

In addition to medical therapy, surgical debridement is usually required and may involve exenteration (ie, surgical removal of the entire globe and surrounding structures) in cases of orbital apex involvement. ${ }^{22,30}$

\section{PROGNOSIS}

Invasive aspergillosis carries a significantly worse prognosis than the noninvasive form. Invasion of bone and blood vessels makes surgical access and drug penetration challenging and allows the fungus to spread intracranially. The reported mortality rate associated with invasive aspergillosis is $40 \%$, rising to $50 \%$ when there is central nervous system involvement. ${ }^{34}$

Prognosis is often worsened by initial misdiagnosis owing to presenting features that are largely nonspecific. Initial administration of corticosteroids is also associated with a poorer prognosis as a result of iatrogenic potentiation of the infection. ${ }^{17}$

\section{CASE CONCLUSION}

The patient was started on voriconazole and tapered off prednisone. CT of the chest was performed to investigate the lungs for additional areas of infection and was negative. Oculoplastic surgery was consulted regarding the benefit of right orbital exenteration. Because the infection had already spread to the cavernous sinus, it was determined that exenteration would not improve survival and was therefore deferred.

Three weeks after discharge, the patient continued to experience retro-orbital headaches but rated the pain as $1 / 10$ instead of $10 / 10$, as he had consistently rated it before treatment. His right-sided ptosis was slightly improved, but his right eye blindness, afferent pupillary defect, and complete loss of extraocular movements persisted.

\section{TAKE-HOME POINTS}

- Suspected GCA should be treated immediately with glucocorticoids, but lack of improvement, negative temporal artery biopsy, and normal ESR and CRP should prompt investigation of an alternative diagnosis.

- The orbital apex is the posterior-most end of the orbit and contains bony, neural, and vascular structures.

- Orbital apex syndrome is characterized by involvement of cranial nerves II, III, IV, $\mathrm{VI}$, and $\mathrm{V}_{1}$.

- Cavernous sinus syndrome and superior orbital fissure syndrome present similarly to orbital apex syndrome; specific cranial nerve palsies can help differentiate them on physical examination.

- Etiologies of orbital apex syndrome are numerous but fit into these main categories: inflammatory, infectious, neoplastic, iatrogenic, traumatic, and vascular.

- MRI is the best imaging method for visualizing the orbital apex, but CT is useful when examining bone involvement or if the patient has MRI contraindications.

- Orbital mycoses are most often a result of contiguous spread from the paranasal sinuses and are most commonly caused by aspergillosis or mucormycosis.

- Patients with immunodeficiency are at much greater risk of contracting invasive aspergillosis, which carries a significant risk of mortality that further increases with central nervous system involvement.

- Treatment of invasive aspergillosis involves a combination of antifungal management and surgical debridement.

- Voriconazole monotherapy is the preferred initial medical therapy for invasive sinus aspergillosis, with isavuconazole or lipid formulations of amphotericin B being viable alternatives.

\section{DISCLOSURES}

The authors report no relevant financial relationships which, in the context of their contributions, could be perceived as a conflict of interest. 


\section{REFERENCES}

1. Dasgupta B; Giant Cell Arteritis Guideline Development Group. Concise guidance: diagnosis and management of giant cell arteritis. Clin Med (Lond) 2010; 10(4):381-386. doi:10.7861/clinmedicine.10-4-381

2. Dasgupta B, Borg FA, Hassan N, et al. BSR and BHPR guidelines for the management of giant cell arteritis. Rheumatology (Oxford) 2010; 49(8):1594-1597. doi:10.1093/rheumatology/keq039a

3. Chen JJ, Leavitt JA, Fang C, Crowson CS, Matteson EL, Warrington KJ. Evaluating the incidence of arteritic ischemic optic neuropathy and other causes of vision loss from giant cell arteritis. Ophthalmology 2016; 123(9):1999-2003. doi:10.1016/j.ophtha.2016.05.008

4. Hayreh SS, Zimmerman B. Visual deterioration in giant cell arteritis patients while on high doses of corticosteroid therapy. Ophthalmology 2003; 110(6):1204-1215. doi:10.1016/\$0161-6420(03)00228-8

5. Radiopaedia. Orbital apex (diagram). https://radiopaedia.org/cases/ orbital-apex-diagram-1 ?lang=us. Accessed August 9, 2021.

6. Chen CT, Chen YR. Traumatic superior orbital fissure syndrome: current management. Craniomaxillofac Trauma Reconstr 2010; 3(1):9-16. doi:10.1055/s-0030-1249369

7. Prasad S, Galetta SL. Approach to the patient with acute monocular visual loss. Neurol Clin Pract 2012; 2(1):14-23. doi:10.1212/CPJ.0b013e31824cb084

8. Yeh S, Foroozan R. Orbital apex syndrome. Curr Opin Ophthalmol 2004; 15(6):490-498. doi:10.1097/01.icu.0000144387.12739.9c

9. Aryasit O, Preechawai P, Aui-Aree N. Clinical presentation, aetiology and prognosis of orbital apex syndrome. Orbit 2013; 32(2):91-94. doi:10.3109/01676830.2013.764439

10. Goyal P, Lee S, Gupta N, et al. Orbital apex disorders: imaging findings and management. Neuroradiol J 2018; 31(2):104-125. doi:10.1177/1971400917740361

11. Kuybu O, Dossani RH. Cavernous sinus syndromes. In: StatPearls. Treasure Island, FL: StatPearls Publishing; 2021. https://www.ncbi. nlm.nih.gov/books/NBK532976/. Accessed August 10, 2021.

12. Kubota T, Moritani S. Orbital IgG4-related disease: clinical features and diagnosis. ISRN Rheumatol 2012; 2012:412896. doi:10.5402/2012/412896

13. Nasrazadani D, Quick AK, White WA. An erosive lesion in the orbital apex as the presenting sign of sarcoidosis. Digit J Ophthalmol 2017; 23(1):26-28. doi:10.5693/djo.02.2016.01.001

14. Prado-Ribeiro AC, Luiz AC, Montezuma MA, Mak MP, Santos-Silva AR, Brandão TB. Orbital apex syndrome affecting head and neck cancer patients: a case series. Med Oral Patol Oral Cir Bucal 2017; 22(3):e354-e358. doi:10.4317/medoral.21506

15. Prasad S. A window to the brain: neuro-ophthalmology for the primary care practitioner. Am J Med 2018; 131(2):120-128. doi:10.1016/j.amjmed.2017.10.008

16. Hoxworth JM, Glastonbury CM. Orbital and intracranial complications of acute sinusitis. Neuroimaging Clin N Am 2010; 20(4):511526. doi:10.1016/j.nic.2010.07.004

17. Chakrabarti A, Sharma SC, Chandler J. Epidemiology and pathogenesis of paranasal sinus mycoses. Otolaryngol Head Neck Surg 1992; 107(6 pt 1):745-750. doi:10.1177/019459988910700606.1

18. Słowik M, Biernat MM, Urbaniak-Kujda D, Kapelko-Słowik K, Misiuk-Hojło M. Mycotic infections of the eye. Adv Clin Exp Med 2015; 24(6):1113-1117. doi:10.17219/acem/50572

19. Trief D, Gray ST, Jakobiec FA, et al. Invasive fungal disease of the sinus and orbit: a comparison between mucormycosis and Aspergillus. Br J Ophthalmol 2016; 100(2):184-188.

doi:10.1136/bjophthalmol-2015-306945

20. Kontoyiannis DP, Lionakis MS, Lewis RE, et al. Zygomycosis in a tertiary-care cancer center in the era of Aspergillus-active antifungal therapy: a case-control observational study of 27 recent cases. J Infect Dis 2005; 191(8):1350-1360. doi:10.1086/428780

21. Mody KH, Ali MJ, Vemuganti GK, Nalamada S, Naik MN, Honavar SG. Orbital aspergillosis in immunocompetent patients. Br J Ophthalmol 2014; 98(10):1379-1384. doi:10.1136/bjophthalmol-2013-303763

22. Cho SH, Jin BJ, Lee YS, Paik SS, Ko MK, Yi HJ. Orbital apex syndrome in a patient with sphenoid fungal balls. Clin Exp Otorhinolaryngol 2009; 2(1):52-54. doi:10.3342/ceo.2009.2.1.52

23. Mukherjee B, Raichura ND, Alam MS. Fungal infections of the orbit. Indian J Ophthalmol 2016; 64(5):337-345. doi:10.4103/0301-4738.185588

24. Levin LA, Avery R, Shore JW, Woog JJ, Baker AS. The spectrum of orbital aspergillosis: a clinicopathological review. Surv Ophthalmol 1996; 41(2):142-154. doi:10.1016/s0039-6257(96)80004-x

25. Badakere A, Patil-Chhablani P. Orbital apex syndrome: a review. Eye Brain 2019; 11:63-72. doi:10.2147/EB.S180190

26. Sethi P, Saluja R, Jindal N, Singh V. Invasive aspergillosis in an immunocompetent host. J Oral Maxillofac Pathol 2012; 16(2):297-300. doi:10.4103/0973-029X.99096

27. Peters AT, Spector S, Hsu J, et al. Diagnosis and management of rhinosinusitis: a practice parameter update. Ann Allergy Asthma Immunol 2014; 113(4):347-385. doi:10.1016/j.anai.2014.07.025

28. Kusunoki T, Kase K, Ikeda K. A case of orbital apex syndrome due to Pseudomonas aeruginosa infection. Clin Pract 2011; 1(4):e127. doi:10.4081/cp.2011.e127

29. Garcia-Giraldo AM, Mora BL, Loaiza-Castaño JM, Cedano JA, Rosso F. Invasive fungal infection by Aspergillus flavus in immunocompetent hosts: a case series and literature review. Med Mycol Case Rep 2018; 23:12-15. doi:10.1016/j.mmcr.2018.10.006

30. Patterson TF, Thompson GR 3rd, Denning DW, et al. Practice guidelines for the diagnosis and management of aspergillosis: 2016 update by the Infectious Diseases Society of America. Clin Infect Dis 2016; 63(4):e1-e60. doi:10.1093/cid/ciw326

31. Memar MY, Yekani M, Alizadeh N, Baghi HB. Hyperbaric oxygen therapy: antimicrobial mechanisms and clinical application for infections. Biomed Pharmacother 2019; 109:440-447. doi:10.1016/j.biopha.2018.10.142

32. Lee AS, Lee PWY, Allworth A, Smith T, Sullivan TJ. Orbital mycoses in an adult subtropical population. Eye (Lond) 2020; 34(9):1640 1647. doi:10.1038/s41433-019-0733-3

33. Hirabayashi KE, Kalin-Hajdu E, Brodie FL, Kersten RC, Russell MS, Vagefi MR. Retrobulbar injection of amphotericin B for orbital mucormycosis. Ophthalmic Plast Reconstr Surg 2017; 33(4):e94-e97. doi:10.1097/IOP.0000000000000806

34. Choi HS, Choi JY, Yoon JS, Kim SJ, Lee SY. Clinical characteristics and prognosis of orbital invasive aspergillosis. Ophthalmic Plast Reconstr Surg 2008; 24(6):454-459. doi:10.1097/IOP.0b013e31818c99ff

Address: Craig D. Nielsen, MD, FACP, Department of Internal Medicine G10, Cleveland Clinic, 9500 Euclid Avenue, Cleveland, OH 44195; nielsec@ccf.org 